\title{
14. FUNDAMENTAL SPECTROSCOPIC DATA (DONNÉES SPECTROSCOPIQUES FONDAMENTALES)
}

President: A. H. Cook.

Vice-President: R. H. Garstang.

Organizing Committee: G. Herzberg, W. Lochte-Holtgreven, M.V. Migeotte, Ch. Moore-Sitterly, J. G. Phillips, R. Tousey.

\section{SCIENTIFIC PRIORITIES}

The priorities of work in the fields covered by Commission 14 are determined by the needs of other astronomers, for the aim of Commission 14 is to ensure that data are available by which astronomical observations may be interpreted in physical terms. Thus, there is a continuing need for precise measurements of standard wavelengths so that lines observed in astronomical spectra may be interpreted in terms of spectra of atoms or molecules studied in the laboratory. Until recently, only visible spectra were observed in astronomy, but it may be expected that in the next decade there will be a growing need for standard measurements of frequencies (or wavelengths) of lines in the radio, ultra-violet and X-ray regions of the spectrum.

Transition probabilities and cross sections for various processes are needed to interpret physical conditions in astronomical sources, and here again, it is probable that there will be a growing demand for data relating to transitions at energies corresponding to radio, ultra-violet and X-ray spectra.

Following the XIV General Assembly of the Union, IAU Colloquium No. 8, on Experimental Techniques for Determination of Fundamental Spectroscopic Data was held in the Physics Department of Imperial College, London (1).

\section{REFERENCE}

1. Shore, B. 1971, Q. J. R. Astron. Soc., 12, 48-60.

A. H. $\operatorname{coO} \mathrm{K}$

President of the Commission

COMMITTEE 1: STANDARDS OF WAVELENGTH

\section{The primary standard}

Great progress has been made in the work on stabilizing laser transitions by tuning to sharply defined molecular absorption lines. Several such transitions in the visible and infrared have been investigated, and their wavelengths have been measured with reference to the ${ }^{86} \mathrm{Kr}$ primary standard. For the infrared methane-stabilized helium-neon laser line, Barger and Hall (1) find $\lambda_{\text {yac }}=$ $=33922.31376 \pm 0.00012 \AA$, the error limits being set by the properties of the primary standard. The intercomparison of the different laser lines can be made with much higher precision.

By a remarkable development in frequency measurements it has become possible to compare optical frequencies with that of a caesium beam oscillator, the present standard of time interval. The frequency of the methane-stabilized transition at 3.39 $\mu \mathrm{m}$ was thus determined by Evenson and co-workers, and by combining the result with the wavelength for the same transition they obtain (5) for the velocity of light $c=299792.4562 \pm 0.0011 \mathrm{~km} \mathrm{~s}^{-1}$. The quoted figures have been communicated by Kessler. Similar results have very recently been obtained at the National Research Council, Ottawa and at the National Physical Laboratory, Teddington. This development 